\title{
UN NOUVEAU COLLECTEUR AUTOMATIQUE QUANTITATIF DE FECES DE POISSONS
}

\author{
G. ChOUbert (1), J. DE LA NOUE (2), P. LUQUet (1)
}

(1) Laboratoire de Nutrition et d'Élevage des Poissons, Centre de Recherches Hydrobiologiques, B.P. 3, Saint-Pée-sur-Nivelle, 64310 Ascain, France.

(2) Département de Biologie, Faculté des Sciences et de Génie, Université Laval, SainteFny, Québec G1K 7P4, Canada.

\section{RÉSUMÉ} présenté.

Un appareil automatique de récolte quantitative des fèces de poissons est

L'eau d'évacuation des bacs de poissons est filtrée sur des grilles métalliques. Celles-ci, animées d'un mouvement linéaire, soustraient les fèces retenues à l'action de l'eau. Par un arrêt brusque des grilles, les fèces sont projetées dans un plateau réfrigéré puis sont automatiquement congelées. L'autonomie du système n'est limitée que par la capacité des plateaux collecteurs.

Grâce à la récupération quantitative des fèces, les études de digestibilité des aliments naturels ou artificiels sont possibles par la méthode directe.

De plus, cet appareil peut être utilisé pour des espèces chez lesquelles jusqu'alors on ne pouvait étudier la digestibilité par la méthode de pression abdominale.

Par des modifications appropriées, ce dispositif pourrait être appliqué à la récolte des fèces d'animaux aquatiques d'eau douce ou salée et par suite permettrait la collecte de diverses espèces aquatiques (mollusques, crustacés...)

\section{ABSTRACT}

A method for the automatic collection of fish feces is described. Drainage water from fish tanks is filtered through revolving metallic screens which quickly remove feces from the water and propel them into a refrigerated pan, where they are immediately frozen. Operation of the system requires minimal attention and is not time-consuming.

As indicated by the total collection of an inert tracer, chromic oxide, this method enables quantitative recovery of feces and makes digestibility determinations possible, even by the direct method.

\section{INTRODUCTION}

L'utilisation digestive est un des critères d'appréciation de la qualité des matières premières utilisées dans l'alimentation et de l'efficacité des régimes alimentaires.

Chez le poisson, son étude peut être abordée en utilisant deux méthodes :

- la méthode directe qui implique, outre le relevé complet des quantités d'aliment consommé, la collecte intégrale des fèces excrétées, (TUNISON et al, 1944 ; POST et al., 1965);

- la méthode indirecte qui évite de mesurer les quantités ingérées et surtout ne nécessite pas de récolte quantitative des fèces.

Cette méthode consiste à mesurer la concentration du produit étudié dans les ingesta et les excreta par rapport à celle d'un marqueur non absorbé, l'oxyde de chrome (EDIN, 1918). Sa généralisation est limitée car elle implique un traitement additionnel de l'aliment et de ce fait ne peut être utilisée pour la détermination de l'utilisation digestive des aliments d'origine naturelle.

L'utilisation de l'une ou de l'autre méthode implique la récupération des fèces. Chez le poisson, celles-ci étant émises dans l'eau, leur constitution peut être modifiée par dissolution ou délitement (POSSOMPES, 1973; POSSOMPES et al., 1975). De plus, la taille et la consistance même des fèces rendent ces problèmes de récupération difficiles. 
POSSOMPES (1973) a passé en revue différentes méthodes de récolte des fèces : siphonnage, filtration, massage abdominal. II ressort que, si cette dernière méthode est classiquement utilisée (SINGH et NOSE, 1967), différentes remarques peuvent être faites à son égard:

- certains poissons y sont réfractaires,

- une seule récolte peut être effectuée par jour,

- la répétabilité est mauvaise.

- le transit est accéléré et entraîne, de ce fait, une imprécision quant aux mesures de digestibilité, l'absorption s'effectuant également au niveau du rectum (SMITH et LOVELL, 1971 ; AUSTRENG, 1978 ; WINDELL et al., 1978).

Afin d'éviter d'une part toute manipulation des poissons, et d'autre part une dissolution et un délitement important, KAUSHIK et LUQUET (1976) ont utilisé un tapis roulant constitué d'une toile à bluter de $1 \mathrm{~mm}$ de vide de maille pour séparer et recueillir les fèces évacuées en continu avec l'eau d'un aquarium de forme cylindro-conique. Cependant, ce dispositif de séparation et de récupération ne donne pas toujours satisfaction selon la consistance des fèces.

Les appareils décrits par OGINO et al. (1974) et CHO et SLINGER (1978) n'éliminent pas non plus les problèmes de délitement des aliments et fèces.

Le collecteur automatique de fèces de CHOUBERT et al.(1979) donne de bons résultats quelles que soient la nature et la consistance des fèces. Cependant, son seul inconvénient réside dans le mouvement de rotation affectant le déplacement des passoires. Ainsi, dans la position extrême, la distance séparant le tuyau d'évacuation de l'aquarium et la passoire est voisine de $11 \mathrm{~cm}$. L'eau tombant de cette hauteur écrase les fèces contre la paroi de la passoire. Il en résulte une perte des fèces à travers les mailles de la passoire, perte pouvant s'élever jusqu'à 6-8\% de la quantité de fèces récupérée.

L'objet de ce présent travail est de présenter un nouvel appareil automatique, simple, de récolte de fèces de poisson qui, quelles que soient la taille et la consistance des fèces, soit quantitatif afin d'utiliser la méthode directe pour l'étude de l'utilisation digestive de tous les types d'aliments.

\section{APPAREILLAGE}

\section{- Principe}

L'eau d'évacuation des aquariums contenant les poissons passe à travers des grilles métalliques qui retiennent les fèces, les séparent de l'eau et par un mouvement linéaire les soustraient au lessivage.

\section{- Elément constitutif}

Cet appareil se compose d'une chaîne sans fin qui se déplace sur deux pignons entraînés par un moteur. Sur la chaîne sont fixés les rayons qui soutiennent des grilles métalliques.

1) chaîne sans fin : il s'agit d'une chaîne constituée de palettes (1) en nylon de $90,5 \mathrm{~mm} \times 39 \times 4$. L'épaisseur totale du maillon est de $21 \mathrm{~mm}$. Ces palettes sont rattachées entre elles par des étriers (2) en nylon à renfort intérieur (largeur $34 \mathrm{~mm}$, épaisseur $17 \mathrm{~mm}$ ). La chaîne sans fin est constituée de 18 palettes et se déplace sur deux pignons (3) en nylon de 14 dents au pas de 20 . Le diamètre primitif de chaque pignon est de $89,88 \mathrm{~mm}$ et la largeur d'une denture de $15 \mathrm{~mm}$. Chaque pignon est percé en son centre et monté sur un axe (4) de $20 \mathrm{~mm}$ de diamètre.

Un écrou de chaque côté du pignon permet de centrer exactement l'appareil. L'axe repose sur deux roulements à billes (5) étanches $20 \times 35$ (S.K.F. réf. 63002 R S) euxmêmes fixés dans des paliers en tôle.

Toutes les deux palettes sont fixés deux petits supports (6) à $10 \mathrm{~mm}$ du bord extérieur et à $19 \mathrm{~mm}$ des bords latéraux. Ces supports en plastique recoivent les supports de grilles. 
2) grilles : les grilles métalliques (7) en laiton d'un vide de maille de $650 \mu \mathrm{m}$ sont soudées sur les cadres en acier de $120 \mathrm{~mm}$ de long, $59 \mathrm{~mm}$ de large élaborés à partir du fil d'acier de $3 \mathrm{~mm}$ de diamètre. Ces grilles ont une longueur de $100 \mathrm{~mm}$ et une largeur de $56 \mathrm{~mm}$. Ces cadres peuvent être mis en place et démontés instantanément. Pendant une partie du déplacement de la chaîne, les grilles reposent les unes sur les autres.

3) moteur (8): Afin d'éviter un lessivage des fèces par l'eau d'évacuation du bac, les grilles métalliques défilent sous l'orifice d'évacuation (9). Ce mouvement est effectué par un moto-réducteur (PARVALUX SD 41 SS) de 1 tour/min. qui assure un déplacement linéaire de $28 \mathrm{~cm} / \mathrm{min}$.

4) butée : La butée a pour rôle d'arrêter brusquement le mouvement des grilles métalliques. En acier recouvert d'un jonc de plastique, afin de ménager les grilles des chocs répétés, cette butée distante d'une longueur égale à celle des cadres se trouve dans un plan parallèle à celui passant par l'axe moteur mais situé $2,5 \mathrm{~cm}$ en-dessous.

\section{FONCTIONNEMENT}

Les grilles métalliques supportées par les cadres reposant sur les palettes défilent sous l'eau d'évacuation du bac à la vitesse de $28 \mathrm{~cm} / \mathrm{min}$. Pendant ce temps, la grille retient les fèces s'il y en a dans l'eau. Dans son déplacement linéaire, la chaîne entraîne les grilles et soustrait les fèces de l'action de l'eau. La durée pendant laquelle les fèces sont sous l'eau est inférieure à 2 secondes. La grille arrive alors à la verticale, ce qui permet à l'eau de s'égoutter puis, déséquilibrée, elle acquiert une vitesse angulaire de $0,26 \mathrm{t} / \mathrm{sec}$. en décrivant un quart de cercle et heurte la butée (énergie cinétique $: 2,215 \times$ $10-2 \mathrm{~J})$. Sous le choc, les fèces tombent dans un plateau (11) situé plus bas. Le temps pendant lequel les fèces sont à l'air ne dépasse pas 26 secondes.

\section{DIsCussion}

Le problème de l'écrasement des fèces par l'eau d'évacuation des bacs observé avec l'autre collecteur de fèces (CHOUBERT et al., 1979) est donc pratiquement résolu du fait de la très petite chute d'eau, d'ailleurs réglable $(1 \mathrm{a} 1,5 \mathrm{~cm})$ nécessaire.

Le caractère réellement quantitatif de cet appareil a été évalué en mesurant le taux de récupération de l'oxyde de chrome selon le protocole décrit précédemment (CHOUBERT et al., 1979). II s'avère que, avec cet appareil, le taux de récupération de l'oxyde de chrome est supérieur à $99 \%$, ce qui permet d'étudier la digestibilité de tous les types d'aliments naturels ou artificiels par la méthode directe.

L'autonomie du système n'étant limitée que par la capacité des plateaux de récupération peut être variable au gré de l'utilisateur, de quelques minutes à plusieurs heures. Ce système évite donc d'être présent en permanence à proximité des bacs pour effectuer les prélèvements de fèces sitôt leur émission.

Les fèces étant très rapidement soustraites de l'action de l'eau (6 à 15 secondes selon que les fèces sont émises en haut ou en bas du bac) montrent ainsi une composition très voisine de celle qu'elles ont à leur émission. Ce bref temps de contact fèces-eau est un avantage considérable de cet appareil sur les autres systèmes de récolte des fèces tels que le siphonnage des bacs ou le "Guelf-system" de CHO et SLINGER (1978), car il est bien établi que les phénomènes de dissolution et délitement atteignent leur maximum dans les dix premières minutes après l'émission des fèces (POSSOMPES, 1973; WINDEL et al., 1978).

Mis au point pour l'étude de la digestibilité chez la truite arc-en-ciel et la carpe commune, ce dispositif de récolte peut être utilisé pour toute autre espèce de poisson, y compris celles chez lesquelles, jusqu'alors, toute étude de la digestibilité était impossible (poissons plats...) par la méthode de pression abdominale.

Enfin, cet appareil, couplé à un système de plateau tournant supportant les plateaux de récupération, permet, par la récupération quantitative de fèces, l'étude du transit gastro-intestinal. 


\section{BIBLIOGRAPHIE}

AUSTRENG E., 1978. Digestibility determination in fish using chromic oxide marking and analysis of contents from different segments of gastro-intestinal tract. Aquaculture, 13, 265-272.

CHO C.Y., SLINGER S.J., 1978. Significance of digestibility measurement in formulation of feeds for rainbow trout. EIFAC - FAO. Symp. E-6O. $15 \mathrm{pp}$.

CHOUBERT G., DE LA NOUE J., LUQUET P., 1979. Continuous quantitative automatic collector for fish feces. Prog. Fish-Cult., 41, 64-67.

EDIN H., 1918. Orienterande försök över användbarheten av en pa "led-Kroppsprincipen" grundad metod att bestämma en foderblandnings smältbarhed. Médd/ Cent. Anst. Fors Väs Jordbarom-râd. (Stockholm) 165, 1-28.

KAUSHIK S., LUQUET P., 1976. Etude de la digestibilité des acides aminés de régimes à base de zéine chez la Truite arc-en-ciel. Ann. Hydrobiol.; 7, 11-19.

OGINO C., KAKINO J., CHEN M.S., 1973. Protein nutrition in fish. II. Determination of metabolic fecal nitrogen and endogenous nitrogen excretions of carp. Bull. Jpn. Soc. Sci. Fish., 39 (5), 519-523.

POSSOMPES B.P., 1973. Influence de la température sur les besoins en protéines, le transit alimentaire et la digestibilité chez la Truite arc-en-ciel, Salmo gairdneri Richardson. Thèse 3ème cycle. Univ. Paris VI, $58 \mathrm{pp}$.

POSSOMPES B.P., BERGOT P., LUQUET P., 1975. Mise au point d'une méthode d'étude du transit gastro-intestinal chez la Truite arc-en-ciel SalmogairdneriRichardson : Influence du nombre de repas, des quantités ingérées et de la température d'acclimatation. Ann. Hydrobiol., 6, 131-143.

POST G., SHANKS W.E., SMITH R.R., 1965. A method for collecting metabolic excretions from fish. Prog. Fish-Cult., 27, 108-111.

SINGH R.P., NOSE T., 1967. Digestibility of carbohydrates in young rainbow trout. Bull. Freshwater. Fish. Res. Lab. (Tokyo), 17, 21-25.

SMITH B.W., LOVELL R.T., 1971. Digestibility of nutrients in semi-purified rations by channel catfish in stainless steel troughs.. Proc. Annu. Conf. Southeast. Assoc. Game Fish Comm., 25, 452-459.

TUNISON A.V., PHILLIPS A.M., SHAFFER H.B., MAXWELL J.M., BROCKWAY D.R., Mc CAY C.M., 1944. The nutrition of trout. Cortland Hatchery Rep. 13. N.Y. Conserv. Dep., Fish. Res. Bull., 6, 21 pp.

WINDELL J.R., FOLTZ J.W., SAROKON J.P., 1978. Methods of fecal collection and nutrient leaching in digestibility studies. Prog. Fish-Cult., 40, 51-55. 


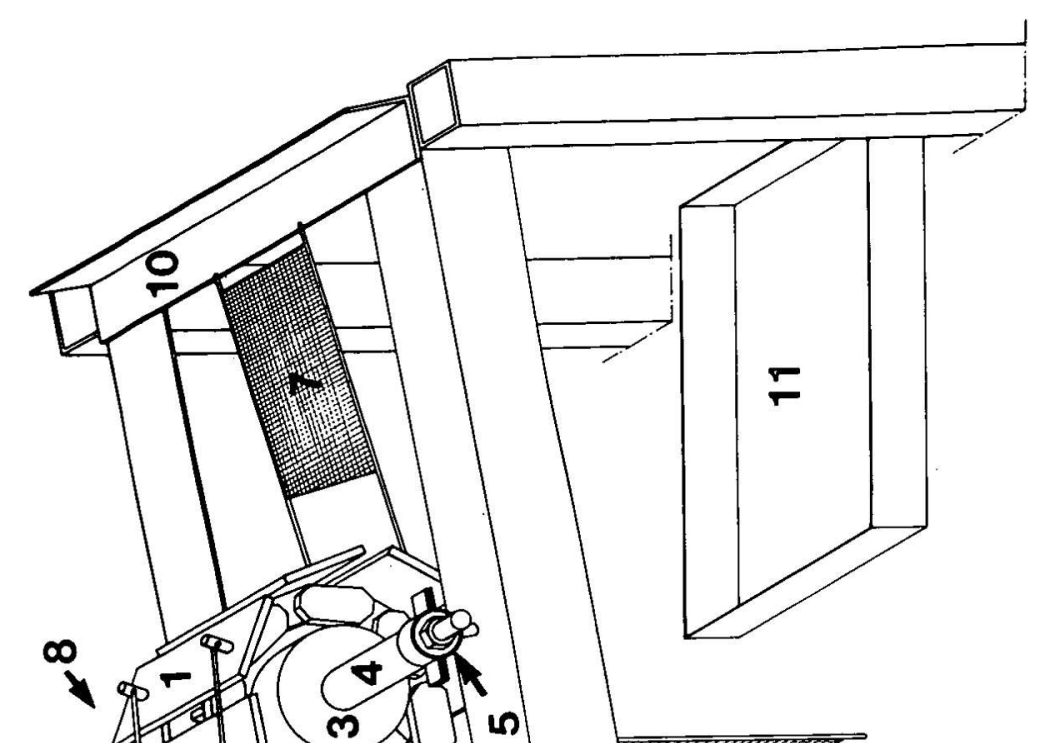

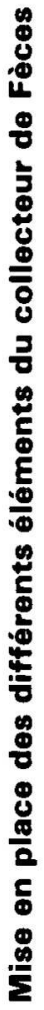

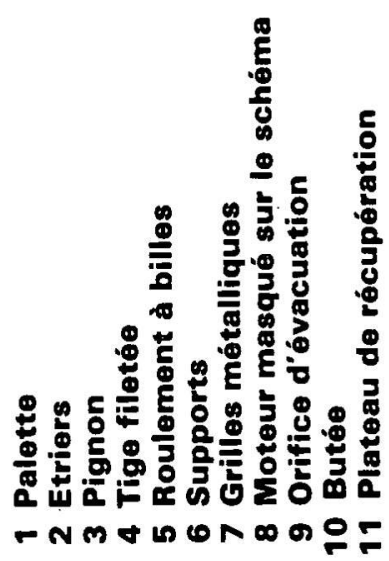

